Fixed Point Theory, 20(2019), No. 2, 507-510

DOI: $10.24193 /$ fpt-ro.2019.2.32

http://www.math.ubbcluj.ro/ nodeacj/sfptcj.html

\title{
ON FIXED POINTS OF SOME FUNCTIONS
}

\author{
ZBIGNIEW GRANDE \\ Institute of Mathematics, Kazimierz Wielki University \\ Plac Weyssenhoffa 11, 85-072 Bydgoszcz, Poland \\ E-mail: grande@ukw.edu.pl
}

Abstract. Let $f:[0,1] \rightarrow[0,1]$ be a Darboux function of Baire class one. Then $f$ has fixed point $x \in[0,1]$, ie. there is a point $x \in[0,1]$ such that $f(x)=x$. So approximate continuity of $f$ implies that $f$ has a fixed point. In this article I investigate when $f$ has a fixed point $x$ satisfying some other conditions (for example $f$ is bilaterally quasicontinuous at $x$ or even continuous at $x$ ).

Key Words and Phrases: Darboux property, Baire class 1, density topologies, quasi-continuity. 2010 Mathematics Subject Classification: 26A05, 26A15, 28A05.

\section{REFERENCES}

[1] J.B. Brown, Almost continuous Darboux functions and Reed's pointwise convergence criteria, Fund Math., 86(1974), 1-17.

[2] A.M. Bruckner, Differentiation of real functions, Lecture Notes in Math. 659, Springer-Verlag, Berlin, 1978.

[3] Z. Grande, An example of a Darboux function having no fixed points, Real Anal. Exch., 28(20022003), No. 2, 375-379.

[4] Z. Grande, On some special notions of approximate quasicontinuity, Real Anal. Exch., 24 (1998-1999), No. 1, 171-184.

[5] S. Kempisty, Sur les fonctions quasicontinues, Fund. Math., 19(1932), 184-197.

[6] T. Neubrunn, Quasi-continuity, Real Anal. Exch., 14(1988/89), No. 2, 259-306.

[7] R.J. O'Malley, Approximately differentiable functions. The $r$ topology, Pacific J. Math., 72 (1977), 207-222.

[8] F.D. Tall, The density topology, Pacific J. Math., 62(1976), 275-284.

Received: December 4, 2017; Accepted: January 15, 2018. 
